Accepted Manuscript

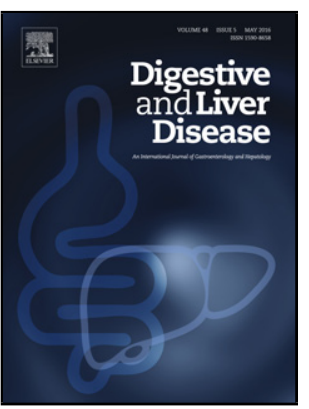

Title: NATURAL HISTORY OF PATIENTS WITH NON CIRRHOTIC PORTAL HYPERTENSION: COMPARISON WITH PATIENTS WITH COMPENSATED CIRRHOSIS

Authors: Stefania Gioia, Silvia Nardelli, Chiara Pasquale, Ilaria Pentassuglio, Valeria Nicoletti, Francesca Aprile, Manuela Merli, Oliviero Riggio

PII: S1590-8658(18)30168-3

DOI: $\quad$ https://doi.org/10.1016/j.dld.2018.01.132

Reference: $\quad$ YDLD 3657

To appear in: $\quad$ Digestive and Liver Disease

Received date: $\quad$ 26-7-2017

Accepted date: $\quad$ 17-1-2018

Please cite this article as: Gioia Stefania, Nardelli Silvia, Pasquale Chiara, Pentassuglio Ilaria, Nicoletti Valeria, Aprile Francesca, Merli Manuela, Riggio Oliviero.NATURAL HISTORY OF PATIENTS WITH NON CIRRHOTIC PORTAL HYPERTENSION: COMPARISON WITH PATIENTS WITH COMPENSATED CIRRHOSIS.Digestive and Liver Disease https://doi.org/10.1016/j.dld.2018.01.132

This is a PDF file of an unedited manuscript that has been accepted for publication. As a service to our customers we are providing this early version of the manuscript. The manuscript will undergo copyediting, typesetting, and review of the resulting proof before it is published in its final form. Please note that during the production process errors may be discovered which could affect the content, and all legal disclaimers that apply to the journal pertain. 


\section{NATURAL HISTORY OF PATIENTS WITH NON CIRRHOTIC PORTAL HYPERTENSION: COMPARISON WITH PATIENTS WITH COMPENSATED CIRRHOSIS}

Short title: Natural history of non-cirrhotic portal hypertension

Gioia Stefania, Nardelli Silvia, Pasquale Chiara, Pentassuglio Ilaria, Nicoletti Valeria, Aprile Francesca, Merli Manuela and Riggio Oliviero.

Dept. of Clinical Medicine, Center for the Diagnosis and Treatment of Portal Hypertension

\section{Address for correspondence:}

Stefania Gioia

Resident in Gastroenterology

Centro di Riferimento per l'Ipertensione Portale.

II Gastroenterologia, Dipartimento di Medicina Clinica

“Sapienza” Università di Roma.

Viale dell’Università 37, 00185 Roma

Phone: $+39(06) 49970463$

Fax: +39(06)49972006

E-mail: stensgioia@ @otmail.com

\section{Key points box:}

- In the patients with non cirrhotic portal hypertension variceal progression is more rapid than in cirrhotic patients. 
- In the patients with non cirrhotic portal hypertension variceal bleeding is more frequent than in cirrhotic patients.

- The patients with INCPH are particularly prompt to develop a portal vein thrombosis

- In patients with non cirrhotic portal hypertension the use of the same strategies used for cirrhotic patients with portal hypertension may not be correct, both in terms of follow-up and treatments

\begin{abstract}
Background. The knowledge of natural history of patients with portal hypertension $(\mathrm{PH})$ not due to cirrhosis is less well known than that of cirrhotic patients.

Aim. To describe the clinical presentation and the outcomes of 89 patients with non-cirrhotic PH (25 with non-cirrhotic portal hypertension, INCPH, and 64 with chronic portal vein thrombosis, PVT) in comparison with 77 patients with Child A cirrhosis.

Methods. The patients were submitted to a standardized clinical, laboratory, ultrasonographic and endoscopic follow-up. Variceal progression, incidence of variceal bleeding, portal vein thrombosis, ascites and survival were recorded.
\end{abstract}

Results. At presentation, the prevalence of varices, variceal bleeding and ascites was similar in the 3 groups. During follow-up, the rate of progression to varices at risk of bleeding $(\mathrm{p}<0.0001)$ and the incidence of first variceal bleeding $(\mathrm{p}=0.02)$ were significantly higher in non-cirrhotic then in cirrhotic patients. A PVT developed in $32 \%$ of INCPH patients and in $18 \%$ of cirrhotics $(\mathrm{p}=0.02)$.

Conclusions. In the patients with non-cirrhotic $\mathrm{PH}$ variceal progression is more rapid and bleeding more frequent than in cirrhotics. Patients with INCPH are particularly prompt to develop PVT. This observational study suggests that the management of patients with non-cirrhotic PH should take into consideration the natural history of portal hypertension in these patients and cannot be simply derived by the observation of cirrhotic patients. 
Abbreviations: NCPH: non-cirrhotic portal hypertension; INCPH: idiopathic non-cirrhotic portal hypertension; PVT: portal vein thrombosis;

Keywords: idiopathic non-cirrhotic portal hypertension, chronic portal vein thrombosis, portal hypertension, natural history

\section{INTRODUCTION}

In the Western world, liver cirrhosis is the most common cause of portal hypertension. However, with the development of imaging technologies, cases of portal hypertension due to chronic portal vein thrombosis (PVT) have been increasingly recognized. In a minority of patients, portal hypertension may also develop in the absence of both cirrhosis and chronic PVT. In these patients the cause of portal hypertension is intrahepatic and histological features involve a wide spectrum of nonspecific changes ranging from sinusoidal dilatation, phlebosclerosis and portal fibrosis to nodular regenerative hyperplasia ${ }^{1}$ in the absence of the histological features of cirrhosis. Recently, the clinical aspects of this infrequent disease have been described and the term idiopathic non cirrhotic portal hypertension (INCPH) has been proposed to facilitate cooperative studies ${ }^{2}$. In the west, the knowledge on the natural history and clinical outcomes of portal hypertension not due to cirrhosis, is based on studies on a limited number of patients either for chronic PVT ${ }^{3-7}$ and $\mathrm{INCPH}^{8-14}$. Because of these limited information, current recommendations for the management of these patients suggest to apply the same guidelines on the treatment of portal hypertension of cirrhotic patients ${ }^{15-16}$.

The aim of the present study was to describe the disease presentation and the incidence of the principal clinical outcomes (variceal progression, incidence of variceal bleeding, portal vein thrombosis and ascites as well as survival) which occurred in a group of 89 patients with portal 
hypertension not due to cirrhosis, in comparison to 77 patients with compensated cirrhosis (Child Pugh A) at the first observation.

\section{PATIENTS AND METHODS}

89 patients with non cirrhotic portal hypertension observed between 1993 and 2016 were included in the study immediately after diagnosis. Sixty-four were affected by chronic portal vein thrombosis (PVT) and 25 by idiopathic non-cirrhotic portal hypertension (INCPH). Portal hypertension was diagnosed on the presence of splenomegaly and oesophageal varices or other portal-systemic collaterals. Portal hypertension was attributed to chronic PVT when imaging techniques (doppler ultrasound and contrast-enhanced CT-scan) showed the presence of portal cavernoma, defined as the development of a network of tortuous collateral vessels bypassing the obstructive area at hepatic hilum. Neoplastic portal vein obstruction was excluded on the basis of imaging studies and evidence of no neoplastic disease. Cirrhosis was excluded on the absence of causes of chronic liver diseases, normal liver protein synthetic capacity, absence of advanced fibrosis on liver elastometry and liver biopsy in selected cases. In addition, liver biopsy was also performed in 13 patients with PVT and no known risk factor for the development of PVT, in order to exclude cirrhosis or obliterative portal venopathy ${ }^{15}$. Moreover, none of the patients classified as affected by portal hypertension secondary to PVT had a previous diagnosis of INCPH.

Portal hypertension was attributed to idiopathic non-cirrhotic portal hypertension (INCPH) after having excluded cirrhosis by liver biopsy and portal and hepatic veins obstruction at Doppler Ultrasound and CT-scan. Other causes of liver disease (chronic viral hepatitis, alcoholic liver disease, non alcoholic steatohepatitis, autoimmune hepatitis, hemochromatosis and Wilson disease) were also excluded by a complete diagnostic clinical and laboratory workout.

Seventy-seven patients with cirrhosis due to HCV infection and belonging to Child-Pugh class A were enrolled as control. The diagnosis of liver cirrhosis was based on clinical, laboratory and 
histological features. No cirrhotic patients received any direct acting antiviral drugs, which were not available during the study period.

\section{Patients initial evaluation and follow-up}

Data collection of each patient started at the moment of first observation. The initial manifestation leading to the diagnostic workout were upper gastrointestinal bleeding due to oesophageal varices in 32 patients, evidence of oesophageal varices at upper endoscopy in 22 patients, evidence of portal cavernoma or portosystemic collaterals in 23 patients and splenomegaly/hypersplenism in 12 patients.

All the patients were evaluated and followed by the same medical team. At the time of the first evaluation, a complete physical examination and routine serum analyses were performed in all patients; their detailed medical history was collected, and their symptoms and signs recorded. For the patients with INCPH and PVT the diagnostic work-up included a systematic screening for any underlying prothrombotic disorder due to hereditary thrombophilia (factor $\mathrm{V}$ Leiden mutation, prothrombin gene mutation, and inherited deficiencies for protein $\mathrm{C}$, protein $\mathrm{S}$ and antithrombin and acquired hypercoagulability (i.e., myeloproliferative disorders, anti-cardiolipin antibodies, lupus anticoagulant antibodies). The screening for thrombophilia was carried out by a referral haematological centre. Since the study of inherited deficiencies in patients with chronic liver disorders is hard, in our study we considered only the patients with isolated protein $\mathrm{C}$ or $\mathrm{S}$ deficiency in which a familial study was after performed. Myeloproliferative diseases were screened by the detection of Janus tyrosine kinase-2 gene (JAK-2 V617F) mutation and confirmed by a bone marrow biopsy in all patients. Bone marrow biopsy was also performed in the patients without JAK2 V617F mutation but with phenotypic features strongly suggestive for a myeloproliferative disease. The patients affected by myeloproliferative neoplasm were regularly followed-up by a haematologist and treated with specific therapy when necessary. 
All patients with PVT were submitted to oral anticoagulation targeting the INR between 2 and 3 . Prophylactic anticoagulation was not proposed in the patients with INCPH or cirrhosis.

An upper endoscopy was carried out in all the patients and oesophageal varices were graded and reported as small or large (medium and large varices were grouped together) ${ }^{17,18}$. Longitudinal diameters of spleen and liver, portal vein diameter, portal vein thrombosis and presence of ascites were studied through Doppler-sonography and recorded.

All patients were regularly followed up with clinical, laboratory and ultrasound evaluation every six months. In case of appearance of hepatic nodules at ultrasonography, the patients were submitted to a second level diagnostic work up for the diagnosis of hepatocellular carcinoma, which included the liver biopsy if necessary. Endoscopic surveillance was made every 12 months, until the evidence of varices at risk of bleeding needing prophylaxis. All episodes of variceal bleeding, ascites or new thrombotic events occurring during the follow-up were recorded. The patients were followed for a mean of $8.4 \pm 15.3$ years.

The purpose of the study was clearly explained to all the patients before obtaining their written informed consent. The "Sapienza" University of Rome Ethical Committee approved the collection of data of the patients for prognostic studies (Rif.1720/01.10.09).

\section{Statistical analysis}

Data are expressed as mean $\pm \mathrm{SD}$, unless specified otherwise. Comparison between 2 groups was performed by chi square test or unpaired Student $t$ test, when appropriate. Comparison between 3 groups was performed by analysis of variance (ANOVA) and by Newman Keuls multiple comparison post hoc analysis. The occurrence of large varices, variceal bleeding, thrombosis, ascites as well as the cumulative survival rate was described by Kaplan-Meier plots. In order to test the independent correlation, adjusted for the other variables, between the diseases (cirrhosis vs non-cirrhotic portal hypertension) and each clinical outcome, we included the variables unequally 
distributed (age, haemoglobin and disease) into a multivariate Cox Regression analysis. The Number Cruncher Statistical System (NCSS) was used for all computations.

\section{RESULTS}

\section{Clinical presentation}

The clinical, biochemical and ultrasonographic features of the patients at entry are summarized in Table 1. Gender distribution was similar in the 3 groups, while age was significantly higher in patients with Child A cirrhosis compared to the other two groups. Bilirubin, ALT, albumin and cholinesterase were similar in the 3 groups while alkaline phosphatase was significant higher in patients with chronic PVT, likely due to the presence of hypertensive biliopathy in some patients. Platelets counts were also higher in the PVT group, likely due to presence of myeloproliferative diseases in $40 \%$ of the patients. Signs of portal hypertension (oesophageal varices, variceal bleeding, ascites, splenomegaly) were equally distributed in the three groups of patients. Spleen diameter was significant larger in patients with INCPH than in the other two groups. Portal vein diameter was larger in INCPH than in cirrhotic patients, while it was not measurable in the group of patients with chronic PVT. A portosystemic shunt was detected in 10 patients with non cirrhotic portal hypertension. The shunt was spleno-renal in 5 patients, gastro-renal in 4 and spleno-gastrorenal in one patient). Presence and size of varices were determined at the time of the first observation of the patients. The number of patients with previous bleeding and therapeutic variceal banding was 10 in the Child A cirrhosis group, 3 in the INCPH group and 8 in the PVT group. The size of varices was similar in the 3 groups, as well the proportion of patients with previous variceal bleeding and previous endoscopic treatment at the first observation. The presence of ascites was also similar in the 3 groups.

The presence of thrombophilic conditions was searched in the patients with non-cirrhotic portal hypertension only. A thrombophilia was found in 6 patients with INCPH (24\%) and in 42 patients with chronic PVT (66\%). The different kind of prothrombotic disorders is reported in Table 2. Four 
patients with INCPH (16\%) and 16 patients with PVT (25\%) had at least one prothrombotic disorders while chronic myeloproliferative neoplasms were diagnosed in 2 patients with INCPH and in 26 patients with PVT.

Moreover, other 8 patients were classified as having significant extrahepatic comorbidities or diseases known to be associated to INCPH. In particular 3 patients were treated with oxaliplatin chemotherapy for colorectal cancer, 3 patients were affected by common immune variable deficiency (CVID), one was affected by coeliac disease and one patient was HIV-positive.

\section{Clinical outcomes during follow-up}

\section{Development of varices at risk of bleeding and incidence of variceal bleeding}

The development of large varices was studied only in the subgroup of patients and with no or small varices at first endoscopy and in patients without a history of previous variceal bleeding. In 3 patients the first endoscopy was not available so they were not considered for variceal progression. In the 25 patients with INCPH, 11 had low risk varices (Absent=8; Small=3); in the 64 patients with PVT, 27 patients had low risk varices (Absent=15; Small=12). Finally, in the group of Child $A$ cirrhosis $(n=77), 40$ patients had low risk varices (Absent=24; Small=16). None of these patients received drugs to reduce portal hypertension.

Four patients with INCPH, thirteen with chronic PVT and twelve with compensated cirrhosis developed large varices during follow-up. The Kaplan-Maier curve showing the patients free of large varices during the follow up is reported in figure 1 in which, given the small number, the patients with INCPH and chronic PVT were pooled. The results are thus presented comparing the group of patients with Child $A$ cirrhosis (n=40) with the group of patients with portal hypertension not due to cirrhosis $(n=38)$ and indicated in the figure as non-cirrhotic portal hypertension (NCPH). In the patients with non-cirrhotic portal hypertension, the rate of progression was more rapid than that observed in the patients with cirrhosis ( $\mathrm{p}<0.0001$, log rank test); (Figure 1), HR=5.6 (95\% CI, 2.2-14.1) at Cox multiple regression analysis. The difference between patients with cirrhotic and 
$\mathrm{NCPH}$ remains significant also if the patients are stratified according to the variceal size at first endoscopy (with no varices or with small varices) as reported in Figure 2, Panel A and B.

The incidence of first variceal bleeding during the follow-up was studied in the 109 patients without variceal bleeding at first observation.

Of these 109 patients, 78 were without varices at risk of bleeding at the first observation. These 78 patients were submitted to upper endoscopy every 12 months. The remaining 31 patients with varices at risk of bleeding were submitted to a treatment to prevent the first bleeding (primary prophylaxis) according to the guidelines for the management of portal hypertension in cirrhotic patients ${ }^{16}$, which included the use of non-selective beta-blockers (in 19 patients), carvedilol (in 5 patients), and endoscopic banding (in 5 patients). In the two remaining patients (both with INCPH), TIPS was performed in order to prevent the first variceal bleeding. These two patients had large varices not responding to band ligation and needed for oral anticoagulation because of the development of thrombosis.

In patients with gastrointestinal bleeding upper endoscopy and US was performed immediately. Variceal haemorrhage occurred in four patients with INCPH, in 8 with chronic PVT and in 9 patients with cirrhosis. As before, the patients with portal hypertension not due to cirrhosis were pooled. Variceal bleeding was lower in patients with Child A cirrhosis than in patients with noncirrhotic portal hypertension ( $\mathrm{p}=0.02, \log$ rank test) (Figure 3) $H R=4.1$ (95\% CI, 1.5-10.8) at Cox multiple regression analysis. No other causes of bleeding were observed.

A Doppler Ultrasound was routinely performed in all bleeding patients in order to check for the development of PVT. In no patients we found a relationship between thrombosis/re-thrombosis development and variceal bleeding.

After bleeding, all the patients were submitted to secondary prophylaxis with the combination of band ligation and beta-blockers or TIPS according to the indication for the management of cirrhotic patients. TIPS was necessary for the prophylaxis of variceal rebleeding in 4 with INCPH, 3 patients with chronic PVT and only 1 patient with cirrhosis. 


\section{Portal vein thrombosis}

During the follow up, portal vein thrombosis occurred in eight patients with INCPH (32\%) and in 14 patients with Child $A$ cirrhosis (18\%); among the eight INCPH patients who developed portal vein thrombosis, six had a prothrombotic state and one was HIV positive. The incidence of portal vein thrombosis was significantly higher in patients with INCPH than in patients with compensated cirrhosis ( $\mathrm{p}=0.02, \log$ rank test) (Figure 4). A new thrombosis of the splanchnic area occurred in 13 (20.3\%) patients with chronic PVT. Among the thirteen patients with a new thrombotic event, in five oral anticoagulant therapy was temporarily stopped and temporarily shifted to LMWH because of the development of large varices with need of banding or because of variceal bleeding. Moreover, 6 patients had an underlying myeloproliferative neoplasm, which represented $23 \%$ of the patients with MPN. In these 6 patients a trend to higher gamma-GT levels than in those with MPN without re-thrombosis was observed $(186 \pm 177$ vs $68.5 \pm 61 ; p=0.03)$.

\section{Ascites and survival}

During the follow up, ascites occurred in one INCPH patient, in four patients with PVT and in nine with cirrhosis class A Child-Pugh with an incidence rate higher in patients with cirrhosis than in patients with $\mathrm{NCPH}(\mathrm{p}=0.004, \log$ rank test) $H R=0.08$ (95\% CI, 0.01-0.57) at Cox multiple regression analysis. None of the patients developed a hepatocellular carcinoma.

During follow-up, 12 patients with cirrhosis and 5 patients with PVT died. Only one patient with INCPH died during follow-up because of opportunistic encephalitis in HIV positive patient. No significant difference in survival was found between the three groups of patients (Figure 5).

\section{DISCUSSION}

Current recommendations on vascular liver disease ${ }^{15-16}$ suggest to manage the patients with portal hypertension not due to cirrhosis according to the guidelines elaborated for cirrhotic patients. Accordingly, for example, the timing for the endoscopic surveillance should be the same of 
cirrhotics. However, in cirrhotic patients this timing is based on the knowledge of the rate of progression of varices, which could be quite different in patients affected by a vascular liver disease. These "by analogy" recommendations are mainly due to the paucity of studies on the natural history of non cirrhotic portal hypertension in which a direct comparison of the main clinical outcomes occurring in patients with portal hypertension due or not due to cirrhosis 19,20 is often lacking. This makes these recommendations at least arguable.

To address this problem we reviewed the records of a group of patients with non-cirrhotic portal hypertension prospectively followed up according to a standardized protocol in our centre dedicated to the management of portal hypertension.

The aim of our study was to determine the main presentation of non-cirrhotic portal hypertension and to describe the incidence of the most relevant clinical outcomes in comparison with those observed in a group of cirrhotic patients followed up according to the same protocol. For this aim all consecutive patients affected by chronic PVT or by INCPH were included. As control group, a cohort of Child-Pugh class A HCV positive cirrhotic patients was enrolled and followed up. The choice to include only patients with compensated cirrhosis due to HCV was made to have a control group with limited liver damage and with a natural history and disease progression more stable than that observable in other ethiology, such as alcohol.

At diagnosis, there are no differences between patients with portal hypertension due to or not due to cirrhosis about signs of portal hypertension and liver function (Table 1). Compared to the other groups, the patients with INCPH had a spleen and portal vein diameter significantly higher than those of patients with cirrhosis. For the analysis of most clinical variables the patients with chronic PVT and INCPH were pooled in the group of non cirrhotic portal hypertension.

In patients with non cirrhotic portal hypertension the rate of development of varices at risk of bleeding was significant higher than in patients with cirrhosis independently on the size of varices at the first endoscopy (Figure 1 and Figure 2, Panel A and B). This observation may at least 
question the recommendation of repeating the endoscopy at the same time in patients with cirrhotic and non-cirrhotic portal hypertension. For example, according to the suggested guidelines for cirrhosis, a patient with no varices at the first endoscopy should be submitted to the next endoscopy after 2 or 3 years. However, at that time about $20 \%$ of the patients with NCPH already developed varices at risk (Figure 2, Panel A). The same occurs in patients with small varices at enrolment, in whom the next endoscopic control should be repeated one year later. Again, at that time, apparently, about $30 \%$ of the patients with NCPH already developed varices at risk. As the time of endoscopy defines the start of the prophylactic treatment, the recommendation to repeat endoscopy with the same schedule used in cirrhotic patients seems to be unjustified. The higher rate of variceal bleeding observed during the follow-up in patients with $\mathrm{NCPH}$ compared to cirrhosis, seems to further support the suggestion of a more rapidly progression of varices in this kind of patients.

Another relevant difference between cirrhotic and non-cirrhotic patients is represented by the incidence of portal vein thrombosis, which is expectedly higher in the patients with chronic PVT. Our study confirm that the development of portal vein thrombosis is a common complication also of $\mathrm{INCPH}^{21,22,23}$ and suggests that it occurs more frequently than in patients with Child $A$ cirrhosis. In 6 out of 8 INCPH patients in our series, it occurred in the presence of an underlying prothrombotic state ${ }^{9}$, suggesting that in patients with INCPH, the slowdown in the flow of the portal vein is a necessary but not sufficient condition to allow the event to occur. This finding confirms the importance and the necessity to make a systematic screening for any underlying prothrombotic disorder in patients with INCPH and support to start anticoagulation at least in those with a prothrombotic state ${ }^{12,24,25}$.

However, being the gastrointestinal bleeding the main complication of INCPH and the role of thrombophilia in its pathogenesis still unclear, the generalized use of a potentially dangerous treatment such as anticoagulation remains uncertain, especially because a clear demonstration of the worsening of the natural history the disease after the development of a PVT is lacking. 
Finally, we observed a recurrence of the splanchnic thrombosis in 13 patients with chronic PVT despite oral anticoagulation. Six of these 13 patients were affected by a MPN and 5 were temporarily shifted to LMWH because of variceal bleeding or progression needing endoscopic banding. This figure is similar to that reported in other series ${ }^{25-26}$. This observation suggests to avoid unnecessary suspension of oral anticoagulation, in all patients and to optimize the treatment of the underlining disease in the patients with splanchnic thrombosis and MPN. Possible additional treatments in these latter patients are possible but never supported by evidences. Also of interest is the observation of higher Gamma-GT levels in the patients with MPN who developed a rethrombosis. Gamma-GT has been shown to be an independent biomarker of PVT in MPN patients ${ }^{27}$ without liver damage or portal hypertension. In patients with portal cavernoma, high gamma-GT may be related to portal biliopathy and/or the underlining liver damage. More data are needed to establish if gamma-GT may be considered an independent biomarker of PVT also in this kind of patients, and if it may contribute to the identification of patients particularly prompt to this complication, eventually leading to changes in their management.

However, the need to submit the patients with non cirrhotic portal hypertension to anticoagulation stress the importance of the endoscopic follow up in this kind of patients.

Differently from the development of varices, the incidence of ascites is significantly lower in non cirrhotic patients while the overall survival resulted similar in patients with non cirrhotic portal hypertension and in patients with cirrhosis.

In conclusion, more studies are needed to describe the natural history of patients with both INCPH and chronic PVT, with the aim of identifying clinically significant outcomes and the best management strategy. The infrequent occurrence of these diseases might heavily limit the possibility of carrying on randomized controlled trials. However, in these patients the use of the same strategies used for cirrhotic patients with portal hypertension may not be correct, both in terms of follow-up and treatments. The results of the present study, although obtained on a limited group of patients support the above conclusion. Cooperative multicentre studies, with an accurate patients' 
selection to limit their heterogeneity, are probably the best tool to attempt an answer to the many questions raised when dealing with such a complex disorders.

Disclosures: the authors declare that no conflict of interest exists concerning this paper

\section{Specific author contributions:}

Stefania Gioia: acquisition of data, analysis and interpretation of data; manuscript preparation.

Silvia Nardelli: acquisition of data

Chiara Pasquale: acquisition of data

Ilaria Pentassuglio: acquisition of data

Valeria Nicoletti: acquisition of data

Francesca Aprile: acquisition of data

Manuela Merli: analysis and interpretation of data

Oliviero Riggio: study concept and design, analysis and interpretation of data; manuscript preparation; final drafting of the manuscript; study supervision.

Financial support: no financial support

\section{Conflict of interest}

The authors declare that no conflict of interest exists concerning this paper. 


\section{REFERENCES}

1) Verheij J, Schouten JN, Komuta M, Nevens F, Hansen BE, Janssen HL, Roskams T. Histological features in western patients with idiopathic non-cirrhotic portal hypertension. Histopathology. 2013 Jun; 62(7):1083-91

2) Schouten JN, Garcia-Pagan JC, Valla D, and Janssen H. Idiopathic non-cirrhotic portal hypertension. Hepatology 2011; 54: 1071-81.

3) Condat B, Valla D. Nonmalignant portal vein thrombosis in adults. Nat Clin Pract Gastroenterol Hepatol 2006; 3: 505-515.

4) Valla DC, Condat B. Portal vein thrombosis in adults: pathophysiology, pathogenesis and management. J Hepatol 2000; 32: 865-871.

5) Sobhonslidsuk A, Reddy KR. Portal vein thrombosis: a concise review. Am J Gastroenterol 2002; 97: 535-541.

6) Harmanci O, Bayraktar Y. Portal hypertension due to portal venous thrombosis: etiology and clinical outcome. World J Gastroenterol 2007;13: 2535-2540.

7) Thatipelli MR, Mcbane RD, Hodge DO, Wysokinski WE. Survival and recurrence in patients with splanchnic vein thromboses. Clinical Gastroenterology and Hepatology 2010; 8: 200-205.

8) Villeneuve JP, Huet PM, Joly JG, Marleau D, Cote J, Legare A, et al. Idiopathic portal hypertension. Am J Med 1976;61:459-464.

9) Hillaire S, Bonte E, Denninger MH, Casadevall N, Cadranel JF, Lebrec D et al. Idiopathic non-cirrhotic intrahepatic portal hypertension in the West: a re-evaluation in 28 patients. Gut $2002 ; 51: 275-80$.

10) Eapen CE, Nightingale P, Hubscher SG, Lane PJ, Plant T, Velissaris D, et al. Non-cirrhotic intrahepatic portal hypertension: associated gut diseases and prognostic factors. Dig Dis Sci $2011 ; 56: 227-235$. 
11) Schouten JN, Nevens F, Hansen B et al. Idiopathic non cirrhotic portal hypertension is associated with poor survival: results of a long-term cohort study. Aliment. Pharmacol. Ther. 2012; 35; 1424-1433.

12) Siramolpiwat S, Seijo S, Miquel R, Berzigotti A, Garcia-Criado A, Darnell A, et al. Idiopathic portal hypertension: Natural history and long-term outcome. Hepatology 2014; 59 (6):2276-2285

13) Sarin SK, Kapoor D. Noncirrhotic portal fibrosis: current concepts and management. J Gastr oenterol Hepatol 2002; 17: 526-34.

14) Sarin SK, Kumar A, Chawla YK, Baijal SS, Dhiman RK, Jafri W, et al. Noncirrhotic portal fibrosis/idiopathic portal hypertension: APASL recommendations for diagnosis and treatment. Hepatol Int 2007;1:398: 413

15) EASL Clinical Practice Guidelines: Vascular diseases of the liver. J Hepatol 2015

16) de Franchis R. Expanding consensus in portal hypertension report of the Baveno VI Consensus Workshop: stratifying risk and individualizing care for portal hypertension. J Hepatol 2015; 63:743-752.

17) Merli M, Nicolini G, Angeloni S, Rinaldi V, De Santis A, Merkel C, Attili AF, Riggio O. Incidence and natural history of small esophageal varices in cirrhotic patients. Journal of Hepatology 2003; 38: 266-272.

18) Zoli M, Merkel M, Magalotti D, Gueli C, Grimaldi M, Gatta A, et al. Natural history of cirrhotic patients with small oesophageal varices: a prospective study. Am J Gastroenterol 2000; 95: 503-508.

19) Nevens F, Staessen D, Sciot R, et al. Clinical aspects of incomplete septal cirrhosis in comparison with macronodular cirrhosis. Gastroenterology 1994; 106: 459-63

20) Okuda K, Kono K, Ohnishi K et al. Clinical study of eighty-six cases of idiopathic portal hypertension and comparison with cirrhosis with splenomegaly. Gastroenterology. 1984 Apr; 86(4):600-10. 
21) Schouten JNL, Verheij J and Seijo S. Idiopathic non-cirrhotic portal hypertension: a review. Orphanet Journal of Rare Diseases 2015; 10:67

22) Chang PE, Miquel R, Blanco JL, Laguno M, Bruguera M, Abraldes JG, et al. Idiopathic portal hypertension in patients with HIV infection treated with highly active antiretroviral therapy. Am J Gastroenterol 2009; 104:1707-1714.

23) Matsutani S, Maruyama H, Akiike T, Kobayashi $S$ et Al. Study of portal vein thrombosis in patients with idiopathic portal hypertension in Japan Department of Medicine and Clinical Oncology, Chiba University Graduate School of Medicine, Chiba, Japan. Liver International 2005: 25: 978-983

24) Valla DC. Thrombosis and anticoagulation in liver disease. Hepatology 2008; 47: 13841393.

25) Condat B, Pessione F, Hillaire S, Denninger MH, Guillin MC, Poliquin M, Hadengue A, Erlinger S and Valla D. Current Outcome of Portal Vein Thrombosis in Adults: Risk and Benefit of Anticoagulant Therapy. Gastroenterology 2001; 120: 490-497

26) Spaander M.C.W, Hoekstra J, Hansen B.E et Al. Anticoagulant therapy in patients with noncirrhotic portal vein thrombosis: effect on new thrombotic events and gastrointestinal bleeding . Journal of Thrombosis and Haemostasis 2013; 11: 452-459

27) Görtzen J, Lena M, Hunka MD et Al. $\gamma$-Glutamyl Transferase is an independent biomarker of splanchnic thrombosis in patients with myeloproliferative neoplasm Medicine (Baltimore). 2016 May;95(20):e3355.

\section{FIGURES LEGEND}

Figure 1. Incidence of progression to varices at risk of bleeding (cirrhosis vs non cirrhotic portal hypertension) 


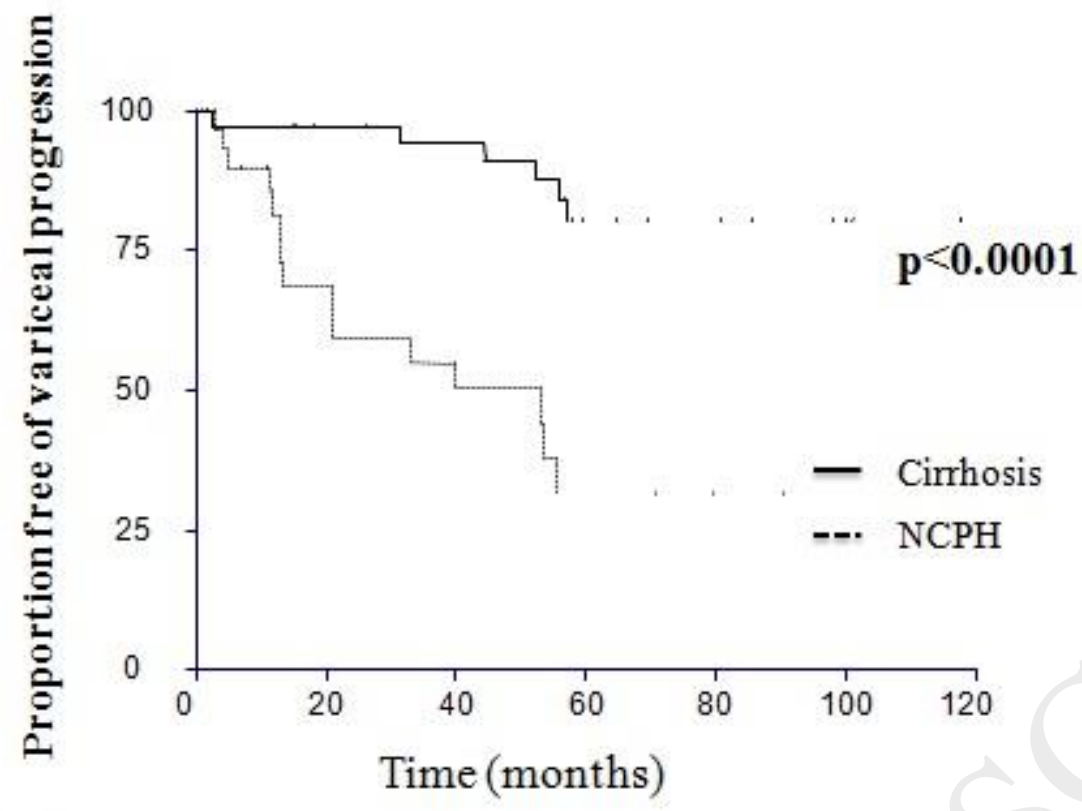

Number at risk: Cirrhosis $\mathrm{NCPH}$

$\begin{array}{rrrrrrr}40 & 33 & 29 & 20 & 18 & 14 & 11 \\ 38 & 15 & 11 & 5 & 3 & 2 & 1\end{array}$


Figure 2. Progression to varices at risk of bleeding (cirrhosis vs non cirrhotic portal hypertension) in patients with no varices (panel A) and in patients with small varices (panel B) at first endoscopy
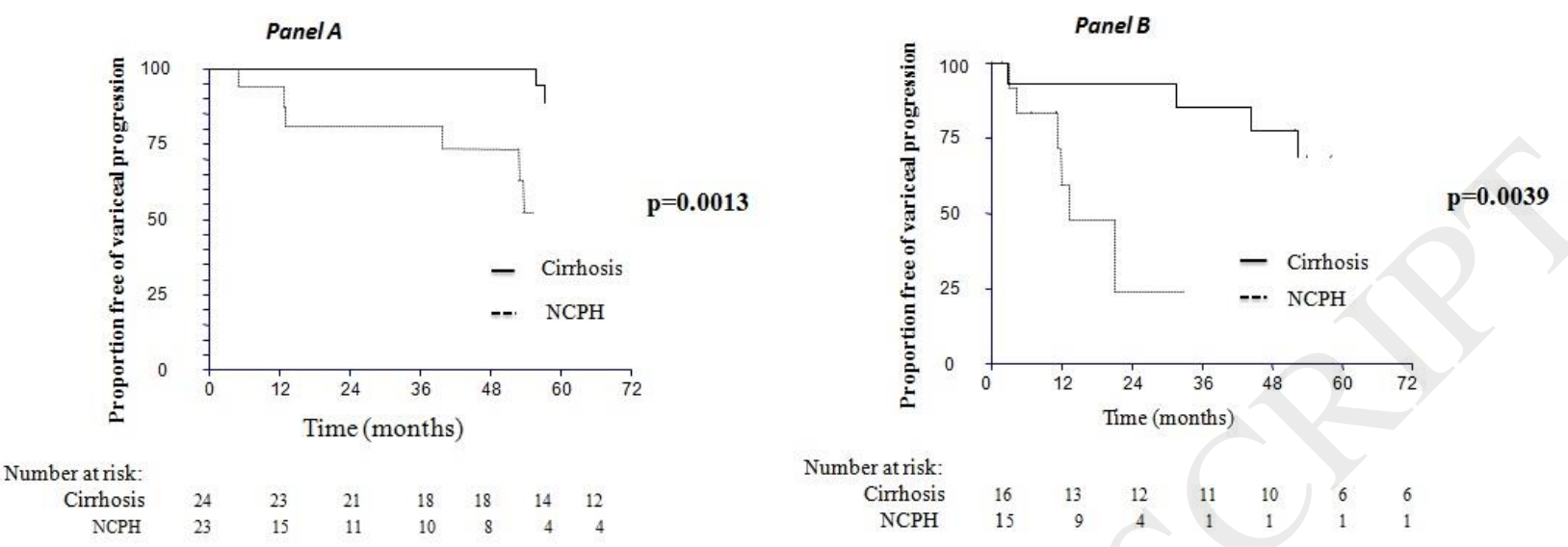

Number at risk:

\begin{tabular}{cccccc|cc} 
Cirrhosis & 16 & 13 & 12 & 11 & 10 & 6 & 6 \\
NCPH & 15 & 9 & 4 & 1 & 1 & 1 & 1
\end{tabular} 
Figure 3. Incidence of variceal bleeding (cirrhosis vs non cirrhotic portal hypertension)

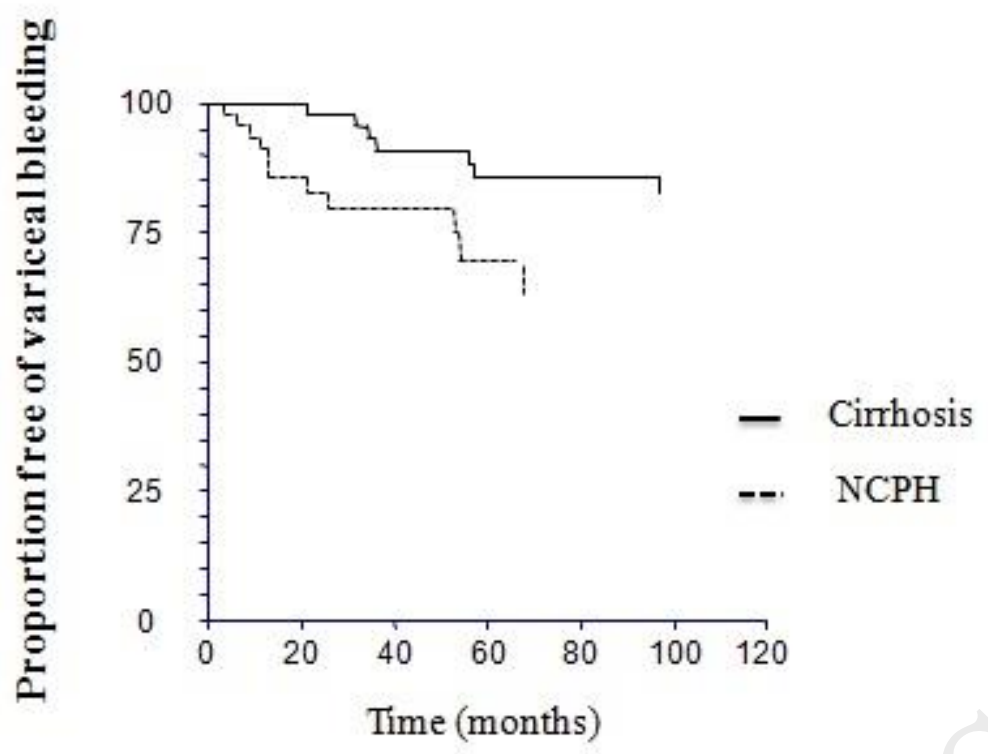

Number at risk:

$\begin{array}{llllllll}\text { Cirrhosis } & 57 & 48 & 38 & 33 & 29 & 25 & 21\end{array}$

$\begin{array}{llllllll}\mathrm{NCPH} & 52 & 29 & 21 & 13 & 8 & 7 & 5\end{array}$


Figure 4. Incidence of portal vein thrombosis in patients with INCPH and in patients with cirrhosis

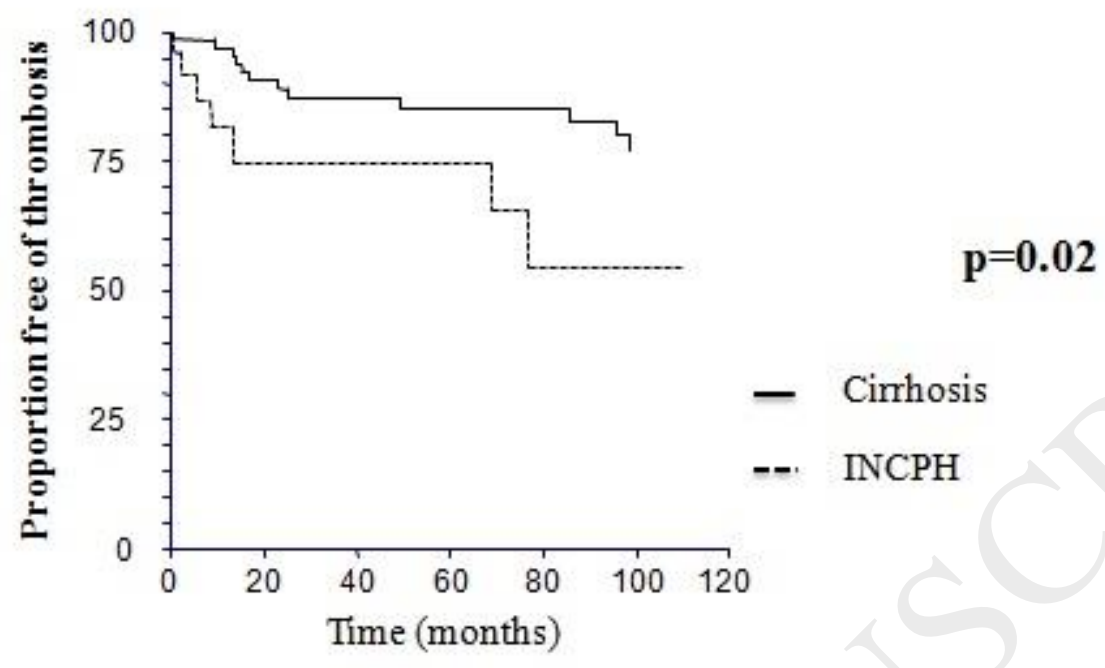

Number at risk:

$\begin{array}{llllllll}\text { Cirrhosis } & 67 & 55 & 45 & 39 & 36 & 26 & 23\end{array}$

$\begin{array}{llllllll}\mathrm{INCPH} & 25 & 11 & 9 & 8 & 5 & 5 & 3\end{array}$


Figure 5. Cumulative survival (cirrhosis vs non cirrhotic portal hypertension)

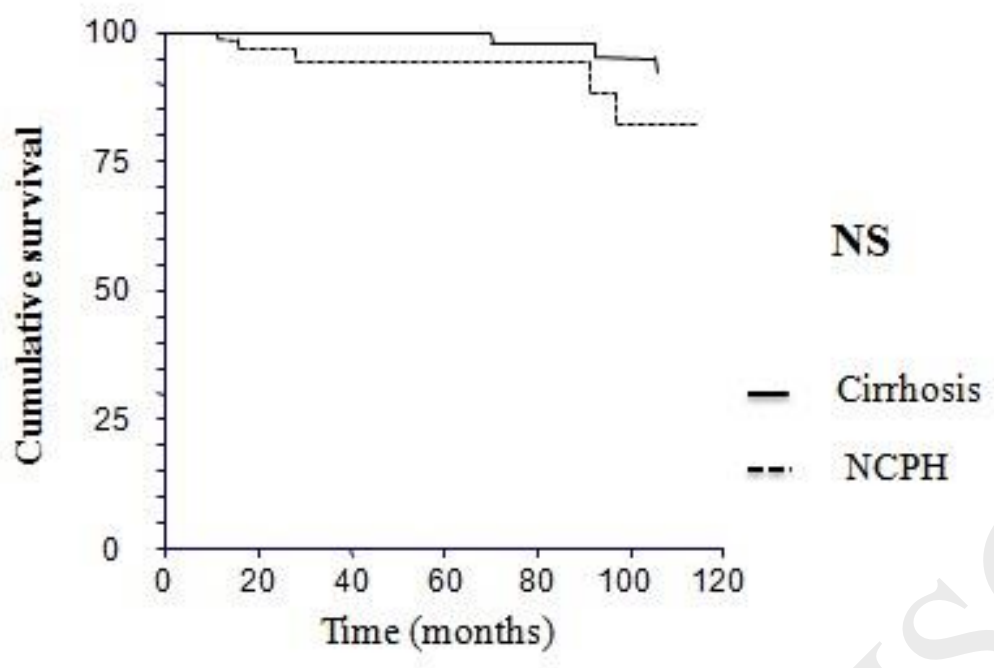

Number at risk:

Cirrhosis

$\begin{array}{lllllll}77 & 63 & 53 & 46 & 40 & 34 & 25\end{array}$

$\mathrm{NCPH}$

$\begin{array}{lllllll}89 & 47 & 33 & 22 & 17 & 13 & 9\end{array}$


Table 1: Characteristics of patients included in the study.

\begin{tabular}{|c|c|c|c|c|}
\hline & $\begin{array}{c}\text { Child A } \\
\text { Cirrhosis }\end{array}$ & INCPH & $\begin{array}{c}\text { Chronic } \\
\text { Portal vein } \\
\text { thrombosis }\end{array}$ & $\begin{array}{l}\text { Grouped } \\
\text { non- } \\
\text { cirrhotic } \\
\text { cohort }\end{array}$ \\
\hline Patients (n) & 77 & 25 & 64 & 89 \\
\hline Age $($ mean \pm SD $)$ & $58.3 \pm 12.5^{*}$ & $45.04 \pm 17$ & $46 \pm 14$ & $45.6 \pm 15.1^{\#}$ \\
\hline $\operatorname{Sex}(M / F)$ & $47 / 30$ & $17 / 8$ & $30 / 34$ & $47 / 42$ \\
\hline Bilirubin (mg/dl) & $1.08 \pm 0.6$ & $1.14 \pm 1.0$ & $1.22 \pm 0.7$ & $1.19 \pm 0.82$ \\
\hline ALT (UI/l) & $71 \pm 121$ & $33.2 \pm 18.8$ & $47 \pm 72$ & $42.7 \pm 61.3$ \\
\hline $\begin{array}{l}\text { Alkaline } \\
\text { phosphatase } \\
\text { (UI/l) }\end{array}$ & $109 \pm 53$ & $138 \pm 77$ & $233 \pm 188^{*}$ & $202 \pm 166^{\#}$ \\
\hline $\begin{array}{l}\text { Ү-glutamyl } \\
\text { transferase (UI/l) }\end{array}$ & $51.9 \pm 33.6$ & $92.9 \pm 117$ & $86 \pm 133$ & $88.4 \pm 127.8$ \\
\hline Albumin (g/dl) & $3.8 \pm 0.5$ & $4.2 \pm 0.5^{*}$ & $3.9 \pm 0.6$ & $4 \pm 0.6$ \\
\hline $\begin{array}{l}\text { Cholinesterase } \\
\text { (UI/l) }\end{array}$ & $7989 \pm 16466$ & $7070 \pm 2517$ & $5966 \pm 2255$ & $6404 \pm 2403$ \\
\hline $\mathrm{Hb}(\mathrm{g} / \mathrm{dl})$ & $12.9 \pm 2.2$ & $12.3 \pm 2.1$ & $12.08 \pm 2.3$ & $12.2 \pm 2.2^{\#}$ \\
\hline Platelet $\left(\times 10^{3} / \mu \mathrm{l}\right)$ & $111 \pm 95$ & $100 \pm 73$ & $290 \pm 240 *$ & $232 \pm 226^{\#}$ \\
\hline $\begin{array}{l}\text { Esophageal } \\
\text { Varices } \\
\text { (absent/small/larg } \\
\text { e) }\end{array}$ & $24 / 22 / 30 \S$ & $8 / 3 / 14$ & $16 / 15 / 31 \S$ & $24 / 18 / 45$ \\
\hline $\begin{array}{l}\text { Previous Variceal } \\
\text { bleeding } \\
(\mathrm{No} / Y e s)\end{array}$ & $52(68 \%) / 25(32 \%$ & $16(64 \%) / 9(36 \%)$ & $\begin{array}{c}41(64 \%) / 23(36 \% \\
)\end{array}$ & $\begin{array}{c}57 \\
(64 \%) / 32(36 \%)\end{array}$ \\
\hline Ascites (No/Yes) & $\begin{array}{c}61(79 \%) / 16(21 \% \\
)\end{array}$ & $20(80 \%) / 5(20 \%)$ & $\begin{array}{c}50(78 \%) / 14(22 \% \\
)\end{array}$ & $\begin{array}{c}70(79 \%) / 19(21 \\
\%)\end{array}$ \\
\hline $\begin{array}{l}\text { Spleen Diameter } \\
(\mathrm{cm})\end{array}$ & $14.6 \pm 2.9$ & $17.5 \pm 5.7 *$ & $14.3 \pm 2.2$ & $15.2 \pm 4.2$ \\
\hline $\begin{array}{l}\text { Portal Vein } \\
\text { Diameter (mm) }\end{array}$ & $12.5 \pm 2.3$ & $14.8 \pm 2.8^{*}$ & ND & \\
\hline
\end{tabular}

Mean $\pm \mathrm{SD}$

$*$ p $<0.05$ by ANOVA and Newman Keuls for the comparison between cirrhosis, INCPH and chronic portal vein thrombosis;

$\# \mathrm{p}<0.05$ by student $\mathrm{t}$ test or $\mathrm{X}^{2}$ for the comparison between cirrhosis and grouped non-cirrhotic cohort 
$\S$ One patient in the cirrhosis group and 2 patients in the chronic PVT group were not submitted to endoscopy at entry because already submitted to variceal rebleeding prophylaxis when first observed;

$\mathrm{ND}=$ not determined. 
Table 2: Thrombophilic disorders observed in patients with portal hypertension not due to cirrhosis.

\begin{tabular}{|c|c|c|}
\hline & $\begin{array}{c}\text { INCPH } \\
(25)\end{array}$ & $\begin{array}{c}\text { Chronic } \\
\text { Portal vein } \\
\text { thrombosis } \\
(64)\end{array}$ \\
\hline \multicolumn{3}{|l|}{ Thrombophilia (n of abnormalities) } \\
\hline Factor V Leiden & 1 & 1 \\
\hline Prothrombin gene mutation & 0 & 1 \\
\hline Protein $\mathrm{C}$ or Protein $\mathrm{S}$ deficiency & 0 & 8 \\
\hline MTHFR mutation & 1 & 7 \\
\hline Anti-phospholipid antibodies & 0 & 8 \\
\hline Lupus anticoagulant antibodies & 2 & 5 \\
\hline $\begin{array}{l}\text { Myeloproliferative neoplasms } \\
\text { (n of patients) } \\
\text { (Polycythemia Vera/ Essential } \\
\text { Thrombocythemia/ Idiopathic myelofibrosis/ } \\
\text { other) }\end{array}$ & $1 / 0 / 1 / 0$ & $5 / 7 / 10 / 4$ \\
\hline
\end{tabular}

